\title{
Consecutive Excretion of Mycobacterium avium Subspecies paratuberculosis in Semen of a Breeding Bull Compared to the Distribution in Feces, Tissue and Blood by IS900 and F57 Quantitative Real-Time PCR and Culture Examinations
}

\author{
Johannes L. KHOL ${ }^{1) *}$, Petr KRALIK $^{2)}$, Iva SLANA ${ }^{2)}$, Vladimir BERAN ${ }^{2)}$, Christine AURICH ${ }^{3)}$, \\ Walter BAUMGARTNER ${ }^{1)}$ and Ivo PAVLIK ${ }^{2)}$ \\ ${ }^{1)}$ Clinic for Ruminants, Department for Farm Animals and Veterinary Public Health, University of Veterinary Medicine Vienna, \\ Veterinärplatz 1, A-1210 Vienna, Austria, ${ }^{2)}$ Veterinary Research Institute Brno, Department of Food and Feed Safety, Hudcova 70, 621- \\ 00 Czech Republic and ${ }^{3)}$ Centre for Artificial Insemination and Embryo Transfer, Department for Companion Animals and Horses, \\ University of Veterinary Medicine Vienna, Veterinärplatz 1, 1210 Vienna, Austria
}

(Received 4 Feburary 2010/Accepted 23 April 2010/Published online in J-STAGE 7 May 2010)

ABSTRACT. Paratuberculosis (Johne's disease) has emerged as one of the most important diseases in cattle. The role of infected bull semen in the spread of infection remains unclear, as the correlation between the amount of excreted Mycobacterium avium subsp. paratuberculosis (semen and feces) and the infection load (blood and tissues) has not been defined. The aim of the present study was to study by culture, and a quantitative real-time polymerase chain reaction, the presence of bacteria in consecutive semen, blood, and fecal samples collected from one infected Piedmont breeding bull during a 380-day period. Five out of seven blood samples and all nine semen samples were positive in the real-time quantitative polymerase chain reaction with $10^{1}$ to $10^{2}$ and $10^{2}$ to $10^{4}$ copies of IS900/F57 per ml, respectively. In all, there were 9 fecal culture positive samples with too numerous to count colony forming units and positive real-time quantitative polymerase chain reactions ranging from $10^{5}$ to $10^{7}$ copies of IS900/F57. After the bull was euthanized, Mycobacterium avium subsp. paratuberculosis was cultured from various parts of the small and large intestines, liver tissue and lymph nodes and from the epididymis and vesicular glands. The results demonstrate a wide extraintestinal distribution of the bacterium and that breeding bulls should be considered a source of paratuberculosis infection due to their contact with other breeding bulls and a high number of heifers and cows through the natural mating process.

KEY WORDS: breeding bull, cattle, Johne's disease, paratuberculosis, semen analysis.

J. Vet. Med. Sci. 72(10): 1283-1288, 2010

Paratuberculosis (Johne's disease) is caused by Mycobacterium avium subspecies paratuberculosis (MAP), which is a slow growing and acid-fast bacillus that is dependent on mycobactin for growth [11]. Although primarily considered a disease of cattle, sheep and goats, all ruminants are susceptible [16, 29]. Cattle are usually infected with $M A P$ as young calves, but heifers and adult animals are also susceptible [34]. Postnatal fecal-oral transmission is the most important route of infection. $M A P$ is shed in the feces, but it can also be found in the colostrum and milk of asymptomatic cows, in turn serving as an additional source of infection [31]. Transmission via semen, by embryo transfer and from wildlife ruminant reservoirs has also been discussed $[3,12$, 27].

Most clinical cases occur between 3 to 6 years of age and young infected cattle generally do not show clinical signs (silent infection). Very few tests are capable of detecting the infection at this stage, although animals may shed infectious organisms into the farm environment during this period $[1,5,21]$. In the advanced clinical stage, cattle become weak and emaciated and later exhibit decreased milk production, severe diarrhea and cachexia. To date, no

\footnotetext{
* Correspondence to: Khol, J. L., Clinic for Ruminants, Department for Farm Animals and Veterinary Public Health, University of Veterinary Medicine Vienna, Veterinärplatz 1, A-1210 Vienna, Austria.

e-mail: johannes.khol@vetmeduni.ac.at
}

treatment strategies have been successful, and the disease ultimately ends in death [11]. During advanced clinical disease, bulls might be infected in various parts of their reproductive organs and may shed $M A P$ into their semen $[3,14$, 15].

Bacterial culture from tissue, feces, milk or semen samples serves as the gold standard for detection of $\operatorname{MAP}[2,22$, 31]. Although specificity is high, the sensitivity of the bacterial culture is low due to the late onset of shedding in feces, milk and semen, intermittent shedding and/or the late occurrence of $M A P$ in regional lymph nodes and other organs $[3,23,30]$. A polymerase chain reaction (PCR) targeted at specific DNA sequences can be used to confirm the results of a culture examination as well as for detection of $M A P$ in feces, tissue, blood and milk samples. A high specificity of $90 \%$ and close to $100 \%$ for quantitative real-time polymerase chain reaction (qPCR) as well as the possibility of obtaining results in less than $24 \mathrm{hr}$ are advantageous attributes of PCR $[22,30]$. A drawback of this technique involves its inability to differentiate between viable and non-viable $M A P$ organisms along with its relatively high price [22].

The first aim of the present study was to determine the presence of $M A P$ in 9 consecutive semen, blood and fecal samples from an infected beef breeding bull by using PCR and culture examinations during a 380-day period. Deter- 
Table 1. Mycobacterium avium subsp. paratuberculosis detection in semen, blood and feces

\begin{tabular}{|c|c|c|c|c|c|c|c|c|c|}
\hline \multirow{2}{*}{ Day ${ }^{a}$} & \multirow{2}{*}{$\begin{array}{c}\text { Weight } \\
(\mathrm{kg})^{\mathrm{b})}\end{array}$} & \multicolumn{2}{|c|}{ Blood } & \multicolumn{3}{|c|}{ Semen } & \multicolumn{3}{|c|}{ Feces } \\
\hline & & IS900 & $F 57^{\mathrm{c})}$ & IS900) & $F 57^{\mathrm{c})}$ & $\mathrm{CFU}^{\mathrm{d})}$ & IS900 & $F 57^{\mathrm{c})}$ & $\mathrm{CFU}^{\mathrm{d})}$ \\
\hline 0 & 645 & $\mathrm{Nt}$ & $\mathrm{Nt}$ & $5.11 \times 10^{3}$ & $7.69 \times 10^{2}$ & - & - & - & TNTC \\
\hline 59 & 657 & $\mathrm{Nt}$ & $\mathrm{Nt}$ & $1.45 \times 10^{4}$ & $4.11 \times 10^{2}$ & - & $3.09 \times 10^{7}$ & $6.70 \times 10^{6}$ & TNTC \\
\hline 118 & 670 & $5.42 \times 10^{1}$ & - & $2.07 \times 10^{2}$ & - & - & $3.67 \times 10^{7}$ & $9.93 \times 10^{6}$ & TNTC \\
\hline 160 & 698 & $1.19 \times 10^{2}$ & - & $1.51 \times 10^{4}$ & $1.28 \times 10^{3}$ & - & $1.09 \times 10^{5}$ & $1.24 \times 10^{5}$ & TNTC \\
\hline 174 & 714 & - & - & $2.47 \times 10^{3}$ & $2.48 \times 10^{2}$ & - & $1.02 \times 10^{7}$ & $4.33 \times 10^{6}$ & TNTC \\
\hline 188 & 714 & $4.54 \times 10^{1}$ & - & $3.06 \times 10^{4}$ & $1.99 \times 10^{3}$ & - & $1.22 \times 10^{7}$ & $4.98 \times 10^{6}$ & TNTC \\
\hline 202 & 744 & $5.42 \times 10^{1}$ & - & $1.45 \times 10^{3}$ & $2.03 \times 10^{2}$ & - & $1.09 \times 10^{7}$ & $5.15 \times 10^{6}$ & TNTC \\
\hline 259 & 745 & $1.93 \times 10^{2}$ & - & $4.06 \times 10^{1}$ & - & - & $2.02 \times 10^{7}$ & $6.18 \times 10^{6}$ & TNTC \\
\hline 380 & 791 & - & - & $2.31 \times 10^{2}$ & - & - & $1.27 \times 10^{7}$ & $8.25 \times 10^{7}$ & TNTC \\
\hline
\end{tabular}

a) Sampling date; b) body weight of the bull at sampling date; c) examined by quantitative real time PCR (values represent the rounded amount of target molecules per $1 \mathrm{~m} l$ of the semen or blood), IS $900=$ quantitative real time PCR for IS 900 target gene, $F 57=$ quantitative real time PCR for $F 57$ target gene; d) examined by HEYM culture examination; $\mathrm{CFU}=$ Colony forming units; TNTC $=$ Too numerous to count CFUs; $-=$ Negative result (target gene not detected); and $\mathrm{Nt}=$ Not tested.

mination of the distribution of $M A P$ in various tissues, including the respiratory, gastrointestinal and reproductive tracts, was the secondary aim of the present study.

\section{MATERIAL AND METHODS}

Sample collection and necropsy: A Piedmont breed bull (2.5 years old) infected with paratuberculosis was housed in the isolation stable of the Clinic for Ruminants of the University of Veterinary Medicine in Vienna. A total of nine consecutive blood, semen and fecal samples were taken (Table 1). The blood samples were obtained under aseptic conditions from the coccygeal vein by using tubes containing Z-Serum Clot Activator (Vacuette ${ }^{\circledR}$, Greiner Bio-One, Kremsmünster, Austria). For quantitative real-time polymerase chain reaction (qPCR), blood samples were taken by using tubes containing $\mathrm{K}_{3}$ EDTA as an anticoagulant (Vacuette $^{\circledR}$, Greiner Bio-One). Fecal samples were taken rectally. Semen samples were collected by electro-ejaculation as described by Palmer [25] after trimming of hairs and cleaning of the prepuce. The examinations were authorized (GZ 68.205/190-BrGT/2004) according to Austrian national legislation BGBL No. 501/1988.

Euthanasia was performed by intravenous injection of $1 \mathrm{~g}$ thiopental-sodium (Thiopental Sandoz ${ }^{\circledR}$, Kundl, Austria) followed by $80 \mathrm{ml}$ T61 (Hoechst Roussel Vet, Wiesbaden, Germany). Necropsy and histology examinations were performed at the University of Veterinary Medicine Vienna. Histology samples were fixed in $7 \%$ neutral buffered formalin, embedded in paraffin, cut into $2-\mu \mathrm{m}$ sections and stained with hematoxylin and eosin (HE). In addition, ZiehlNeelsen (Z-N) acid-fast staining for detection of MAP was performed on selected organs.

Sample testing procedures: All of the semen samples were investigated for volume, gross appearance, $\mathrm{pH}$, semen density (Thoma chamber), and motility (Computer-assisted semen analysis, Sperm Vision, Minitüb, D-Tiefenbach, Germany) according to Pagl et al. [24]. The blood samples were centrifuged, serum was harvested and the Svanovir ${ }^{\mathrm{TM}}$. enzyme linked immunosorbent assay (ELISA, Svanova, Uppsala, Sweden) was performed for detection of specific antigens against $M A P$ according to the manufacturer's instructions. All samples were stored at $-80^{\circ} \mathrm{C}$ until further processing.

Cultivation was performed by using triple slants of Herrold's egg yolk medium (HEYM) as described by Whipple et al. [33] and modified by Pavlik et al. [26]. Approximately $1 \mathrm{~g}$ of tissue specimen was prepared under sterile conditions and homogenized by laboratory blender stomacher (Kleinfeld Labortechnik, Gehrden, Germany). Tissue and fecal (approximately $1 \mathrm{~g}$ of feces) samples were decontaminated using $0.75 \%$ HPC (hexadecylpyridinium chloride: cetylpyridinium chloride, No. 102340 Merck) for 24 and $72 \mathrm{hr}$, respectively. The semen samples were cultured according to Ayele et al. [3]. The cultivation time for all the specimens was 3 months, and positive results were confirmed with qPCR of colonies as described hereunder.

$D N A$ isolation and $q P C R$ : The DNA from semen and blood was isolated by using a modified form of the protocol included in the QIAamp DNA Blood Mini Kit (Qiagen, Hilden, Germany). Briefly, increased volumes of protease $(100 \mu l)$ and AL buffer (400 $\mu l$, both provided with the kit) and $350 \mathrm{mg}$ of $0.1 \mathrm{~mm}$ zirconia/silica beads (Biospec, Bartlesville, OK, U.S.A.) were added to the tubes with $400 \mu l$ of semen or blood. The samples were incubated, while shaking $(1,400 \mathrm{rpm})$, at $56^{\circ} \mathrm{C}$ for $10 \mathrm{~min}$. Subsequently, they were subjected to mechanical homogenization in a bead beater (MagNA Lyser, Roche, Germany) at 6,400 rpm for $60 \mathrm{sec}$. After addition of $400 \mu l$ of $96 \%$ ethanol (Amresco, Inc., Solon, OH, U.S.A.), the supernatant was loaded onto the spin columns. The binding and washing steps were performed according to the manufacturer's instructions. The DNA was eluted using $100 \mu$ of preheated $\left(70^{\circ} \mathrm{C}\right) \mathrm{TE}$ buffer (Amresco, Inc., Solon, OH, U.S.A.) after 5 min of incubation on the column. To improve the DNA yield, the elution was repeated with the same eluate.

The DNA from tissue samples was isolated according to Moravkova et al. [19] by using a commercial DNeasy Blood 
and Tissue Kit (Qiagen) according to the manufacturer's recommendations. The DNA from fecal samples was isolated by using a QIAamp DNA Stool Mini Kit (Qiagen) according to the manufacturer's recommendations.

For detection of $M A P$ from DNA isolated from the semen and blood samples, IS 900 and F57 quantitative real-time PCR duplex assays with cloned internal amplification controls (IAC) were used [30]. Plasmid gradients with cloned IS900 and F57 PCR products were used as quantification standards (Czech Patent Application No. PV2007-295; European patent No. 08466007 [30]).

\section{RESULTS}

Clinical and spermatological examination: Upon the bull's arrival in the clinic, it was in poor general condition with a body weight of $586 \mathrm{~kg}$ (645 kg at first semen collection) and showed intermittent diarrhea. An andrological examination revealed a dense enlargement of the left cauda epididymis, which showed a diameter of approximately 3 $\mathrm{cm}$ and a solid, nonhomogeneous structure upon ultrasonographic examination. During the following months, the bull recovered and reached a body mass of $791 \mathrm{~kg}$ (Table 1). The bull's feces were normal at most times, but short episodes of diarrhea occurred intermittently. At the end of the experiment, the bull began to lose weight again. The bull developed constant diarrhea that led to euthanasia.

The overall quality of the semen samples collected during the study was poor. The volume of the samples varied between 4 and $12 \mathrm{ml}$. Spermatozoa were detected in 5 out of 9 samples only, and the semen density never exceeded 2.8 million per $\mathrm{m} l$. In the last four samples, the semen motility decreased from 72 to $49 \%$ and the membrane integrity decreased from 67 to $39 \%$, respectively. A parallel decrease in the percentage of morphologically normal spermatozoa from 72 to $26 \%$ was found, and the $\mathrm{pH}$ of the samples varied between 6.0 and 7.4.

Gross pathology: Necropsy of the bull revealed gyrification (brain-like folds) of the mucosa of the small intestine, cecum and colon, as well as enlargement of the mesenteric lymph nodes. The left testis and epididymis were enlarged, whereas the right testis, right epididymis and accessory genital glands appeared normal at necropsy.

Histopathology and Ziehl-Neelsen (Z-N) staining: On histopathology examination, infiltration of the mucosa and submucosa layer with high numbers of macrophages and plasma cells were present in the small intestine, cecum and colon. The intestinal lymph nodes showed hyperplasia with large numbers of lymphocytes, as well as focal accumulation of macrophages and giant cells. Focal calcification, dilated seminiferous tubules with a lack of spermatazoa and infiltration with lymphocytes were present in the left testis. The left epididymis showed severe induration with connective tissue, only a few inflammatory cells were detected. Histopathological examination of the right testis showed no pathologic alterations, but the right epididymis was slightly indurated by fibrous connective tissue. The accessory genital glands appeared within normal limits. Furthermore, chronic interstitial nephritis and nonpurulent hepatitis were present, as well as a large number of sarcosporidial cysts in the myocardium without associated significant reaction.

$\mathrm{Z}-\mathrm{N}$ staining revealed large numbers of acid-fast bacilli (AFB) in the samples from the intestine and intestinal lymph nodes, but no AFB were detected in any other tissues. Samples of the jejunum, jejunal lymph nodes, cecum, liver and lymph nodes were positive for $M A P$ on culture. The numbers of cultured $M A P$ were low (Table 2) in the epididymis and vesicular gland. Using IS900- and F57-specific qPCR, high quantities of MAP DNA were detected in all the examined samples from the gastrointestinal tract and in the hepatic lymph nodes (Table 2).

Detection of MAP in the semen, blood and fecal samples:

Table 2. Mycobacterium avium subsp. paratuberculosis detection in tissue samples

\begin{tabular}{|c|c|c|c|c|}
\hline Tissue Origin & Tissue/Organs & IS900 a) & $F 57^{\mathrm{b})}$ & $\mathrm{CFU}^{\mathrm{c})}$ \\
\hline \multirow[t]{2}{*}{ Pulmonary tract } & Lung & $\mathrm{Nt}^{\mathrm{e})}$ & $\mathrm{Nt}$ & $-f^{f}$ \\
\hline & Tracheobronchial Lnn ${ }^{\mathrm{d})}$ & $\mathrm{Nt}$ & $\mathrm{Nt}$ & - \\
\hline \multirow[t]{4}{*}{ Parenchymatous organs } & Liver & $\mathrm{Nt}$ & $\mathrm{Nt}$ & TNTC $^{g)}$ \\
\hline & Liver Lnn & $3.26 \times 109$ & $2.06 \times 109$ & TNTC \\
\hline & Spleen & $\mathrm{Nt}$ & $\mathrm{Nt}$ & - \\
\hline & Kidney & $\mathrm{Nt}$ & $\mathrm{Nt}$ & - \\
\hline \multirow[t]{6}{*}{ Gastrointestinal tract } & Duodenal mucous & $1.17 \times 1011$ & $2.68 \times 1010$ & TNTC \\
\hline & Duodenal Lnn & $\mathrm{Nt}$ & $\mathrm{Nt}$ & - \\
\hline & Jejunal mucous & $1.58 \times 1011$ & $9.77 \times 1010$ & TNTC \\
\hline & Jejunal Lnn & $1.07 \times 109$ & $5.65 \times 108$ & TNTC \\
\hline & Ileocecal valve & $1.23 \times 1010$ & $6.66 \times 109$ & TNTC \\
\hline & Cecum and colon & $8.86 \times 1010$ & $3.35 \times 1010$ & TNTC \\
\hline \multirow[t]{4}{*}{ Genital tract } & Testes & $\mathrm{Nt}$ & $\mathrm{Nt}$ & - \\
\hline & Epididymis & $\mathrm{Nt}$ & $\mathrm{Nt}$ & 1 \\
\hline & Bulbourethral glands & $\mathrm{Nt}$ & $\mathrm{Nt}$ & - \\
\hline & Vesicular glands & $\mathrm{Nt}$ & $\mathrm{Nt}$ & 7 \\
\hline
\end{tabular}

a) Quantitative real time PCR for IS900 target gene; b) quantitative real time PCR for F57 target gene; c) HEYM culture examination, colony forming units; d) lymph nodes; e) not tested; f) negative result (target gene not detected); and g) too numerous to count CFUs. 
All 9 semen samples tested by qPCR for IS 900 were $M A P$ positive. However, only 6 of the 9 semen samples tested positive by using qPCR specific for F57. Conventional HEYM culture did not reveal mycobacteria in any semen specimen. All blood samples were positive for specific antibodies against $M A P$ by ELISA. Five out of seven whole blood samples were positive for $M A P$ in IS 900 qPCR, while none were $M A P$ positive using $F 57$ qPCR. In all fecal samples, intense growth with too numerous to count colonies (TNTC) of $M A P$ was found using conventional HEYM culture. High concentrations $\left(10^{5}-10^{7}\right)$ of $M A P$ DNA were found using IS900 and F57 qPCR in all fecal samples, with the exception of the first sampling, which showed negative results (Table 1). The qPCR results obtained demonstrated that the lowest amount of MAP DNA was found in the blood samples, while the highest amount was found in the fecal samples (Table 1).

\section{DISCUSSION}

Antemortem and post-mortem diagnostics clearly demonstrated clinical paratuberculosis. At necropsy, chronic nephritis, fibrosis of the epididymis and sarcosporidiosis were also found. Monaghan and Hannan [18] reported kidney lesions, including focal nephritis, as a common finding in slaughtered bulls. The additional pathologic conditions found in the presented case are most likely not related to $M A P$ infection but could have been secondary to a general weakness and increased susceptibility of the host due to mycobacterial infection [11]. The same may be true for the semen quality, which was poor in all collected samples. As generalized infections [28], malnutrition and decreased body mass [4] can reduce the sperm quality in bulls, the reduced semen quality in this case is most likely a result of the poor condition of the animal rather than a response to a $M A P$ infection. The lesions of the left testis and epididymis are typical of bacterial inflammation, which was likely unrelated to MAP infection. Although the bull served as a breeding animal, all the above clinical findings were already present at first sampling.

It has been argued that transmission of $M A P$ via semen is unlikely due to the small amount of $M A P$ in the ejaculates of infected bulls [7, 27]. However, Merkal et al. showed that $M A P$ can be found in uterine fluids and walls of cows after artificial contamination of the uterus [17]. In our study, an IS900 qPCR was positive in all the semen samples. In agreement with our study, Ayele et al. [3] revealed MAP in bull semen samples, and Larsen and Kopecky [14] found $M A P$ in the reproductive organs of six bulls, as well as in one semen sample. These authors also showed that MAP can survive beyond the antibiotic treatment and freezing process for semen samples collected for commercial use. Buergelt et al. [7] performed PCR on two semen samples that were collected one year apart from a bull suffering from clinical paratuberculosis. While the first sample gave a positive result, $M A P$ could not be detected in the second sample that was collected one year later.
Quantitative PCR for the insertion sequence IS900 is used as a standard target for detection of $M A P$ and can be found at a level of 14 to 18 copies per genome; however, the target sequence $F 57$ exists as a single copy within the genome [8, 20,32]. Although PCR for F57 is believed to be more specific, its presence of only one copy per genome decreases the sensitivity as compared with IS900 [30]. This could explain the low level of positive results in the semen and blood samples using F57 PCR. As F57 PCR is primarily used for research purposes, we were unable to find articles involving investigations for MAP presence by $F 57$ detection in blood.

The reason for the failure to detect $M A P$ in fecal samples by PCR at day 0 of the presented study, despite a positive culture result, remains unclear. Although not indicated by internal controls, failure of the amplification (wrong negative PCR) or contamination of the fecal sample (wrong positive culture) has to be considered.

$M A P$ could not be cultivated from the semen samples in the presented study. It is possible that negative culture results were caused by rather small numbers of MAP or the loss of viability in bacteria during sample decontamination. Fecal contamination of the semen samples could also be a possible source of positive PCR results, but this seems unlikely because of the measures performed to prevent sample contamination and the consistency of the PCR results. The question of whether the MAP detected in the semen samples by PCR in the present study was viable or not cannot be answered and needs further investigation.

Buergelt and Williams [6] found MAP DNA in the blood of infected cows by using IS900 PCR. Juste et al. [13] also revealed MAP DNA in blood, but found poor agreement between the results of blood PCR and ELISA. The authors assigned it to the fact that $M A P$-loaded phagocytic cells recirculated from the intestinal lymphoid tissue to other lymphoid locations in an early stage of the infection before the occurrence of specific antibodies. In our case, qPCR (IS900) revealed positive results in the blood of all samples, although the bull was in a late stage of the disease, showing clinical signs of paratuberculosis, and was positive for $M A P$ antibody according to ELISA. These results suggest a rather late circulation of $M A P$ in the blood during infection. In both the blood and semen test results, the IS900 qPCR assay demonstrated a higher sensitivity compared with the F57 qPCR assay.

$M A P$ was also isolated from multiple tissues, including testes and other reproductive organs [15]. In addition to the intestine and intestinal lymph nodes, $M A P$ was also found in the hepatic lymph node, epididymis and bulbourethral gland, as reported previously [3, 10, 14, 15]. Repeated oral ingestion or inhalation of contaminated dust particles and aerosols followed by the trapping of $M A P$ by macrophages in the head and lung lymph nodes was suggested by Pavlik et al. [26]. Clarke [9] and Hirsh and Biberstein [11] also assumed a colonization of different organs by $M A P$ in macrophages via blood or the lymphatic system.

It could be concluded that, for the first time, $M A P$ was 
found in all consecutive semen samples collected over a period of more than one year from a breeding bull suffering from paratuberculosis. The presence of $M A P$ in the blood confirms that $M A P$ is not restricted to the intestinal tract and that in the advanced stages of the disease, it can also affect extraintestinal organs via a blood phase. Although no confirmed case of transmission of paratuberculosis to healthy cows via semen from infected bulls has been reported, the finding that some bulls are regularly shedding $M A P$ in their semen should be considered. Because of their direct or indirect (artificial insemination) contact with a high number of potentially susceptible animals, bulls may play a significant role in the transmission of paratuberculosis. Breeding bulls should be tested regularly for this disease in order to reduce potential transmission by this route.

ACKNOWLEDGMENTS. The authors would like to thank the owner and the bull's veterinarian, who generously cooperated with this study. Furthermore, we would like to thank the members of the Institute of Pathology and Forensic Veterinary Medicine, University for Veterinary Medicine Vienna, for their help and support. We would also like to thank Neysan Donnelly (Aberdeen University, Scotland) for the critical linguistic check of the article. This research was partially supported by grants Nos. MZE0002716202 and QH81065 of the Ministry of Agriculture of the Czech Republic, ParaTBTools (FOOD-SB-023106) from the EU and the Ministry of Education, Youth and Sports of the Czech Republic (AdmireVet; Grant No. CZ 1.05/2.1.00/ 01.0006).

\section{REFERENCES}

1. Antognoli, M. C., Garry, F. B., Hirst, H. L., Lombard, J. E., Dennis, M. M., Gould, D. H. and Salman, M. D. 2008. Characterization of Mycobacterium avium subspecies paratuberculosis disseminated infection in dairy cattle and its association with antemortem test results. Vet. Microbiol. 127: 300-308.

2. Ayele, W. Y., Bartos, M., Svastova, P. and Pavlik, I. 2004. Distribution of Mycobacterium avium subsp. paratuberculosis in organs of natural infected bull-calves and breeding bulls. Vet. Microbiol. 103: 209-217.

3. Ayele, W. Y., Machackova, M. and Pavlik, I. 2001. The transmission and impact of paratuberculosis infection in domestic and wild ruminants. Vet. Medicina. 46: 205-224.

4. Barth, A. D. and Waldner, C. L. 2004. Factors affecting breeding soundness classification of beef bulls examined at the Western College of Veterinary Medicine. Can. Vet. J. 43: 274284.

5. Bolton, M. W., Grooms, D. L. and Kaneene, J. B. 2005. Fecal shedding of Mycobacterium avium subsp. paratuberculosis in calves: implication for disease control and management. pp. 596-600. In: Proceedings of 8th Int. Coll. on Parat.

6. Buergelt, C. D., Donovan, G. A. and Williams, J. E. 2004. Identification of Mycobacterium avium subspecies paratuberculosis by polymerase chain reaction in blood and semen of a bull with clinical paratuberculosis. Intern. J. Appl. Res. Vet. Med. 2: 130-134.

7. Buergelt, C. D. and Williams, J. E. 2004. Nested PCR on blood and milk for the detection of Mycobacterium avium subsp. paratuberculosis DNA in clinical and subclinical bovine paratuberculosis. Aust. Vet. J. 82: 497-503.

8. Bull, T., Hermon-Taylor, J., Pavlik, I., El-Zaatari, F. and Tizard, M. 2000. Characterisation of IS 900 loci in Mycobacterium avium subsp. paratuberculosis and development of multiplex PCR typing. Microbiol. 146: 2185-2197.

9. Clarke, C. J. 1997. The pathology and pathogenesis of paratuberculosis in ruminants and other species. J. Comp. Pathol. 116: $217-261$.

10. Glawischnig, W., Awad-Masalmeh, M., Khasabi, D. and Schönbauer, M. 2004. Nachweis von Mycobacterium avium subsp. paratuberculosis im Hoden eines klinisch erkrankten Zuchtstiers (Mycobacterium avium subsp. paratuberculosis in the testis of a subclinically infected breeding bull. Berl. Münch. Tierärztl. Wschr. 117: 136-139 (in German, abstract in English).

11. Hirsh, D. C. and Biberstein, E. L. 2004. Mycobacteria. pp. 223-234. In: Veterinary Microbiology, 2nd ed. (Hirsh, D. C., MacLachlan, N. J. andWalker, R. L. eds.), Blackwell, Oxford.

12. Judge, J., Kyriazakis, I., Greig, A., Allcroft, D. J. and Hitchings, M. R. 2005. Clustering of Mycobacterium avium subsp. paratuberculosis in rabbits and the environment: how hot is a hot spot? Appl. Environ. Microbiol. 71: 6033-6038.

13. Juste, R. A., Garrido, J. M., Geio, M., Elquezabal, N., Aduriz, G., Atxareandio, R. and Sevilla, I. 2005. Comparison of blood polymerase chain reaction and enzyme-linked immunosorbent assay for detection of Mycobacterium avium subsp. paratuberculosis infection in cattle and sheep. J. Vet. Diagn. Invest. 17: 354-359.

14. Larsen, A. B. and Kopecky, K. E. 1970. Mycobacterium paratuberculosis in reproductive organs and semen of bulls. Am. J. Vet. Res. 31: 255-258.

15. Larsen, A. B., Stahlheim, O. H. V., Hughes, D. E., Appel, L. H., Richards, W. D. and Himes, E. M. 1981. Mycobacterium paratuberculosis in the semen and fenital organs of a semendonor bull. J. Am. Vet. Med. Assoc. 179: 169-171.

16. Machackova, M., Svastova, P., Lamka, J., Parmova, I., Liska, V., Smolik, J., Fischer, O. A. and Pavlik, I. 2004. Paratuberculosis in farmed and free-living wild ruminants in the Czech Republic (1999-2001). Vet. Microbiol. 101: 225-234.

17. Merkal, R. S., Miller, J. M., Hintz, A. M. and Bryner, J. H. 1982. Intrauterine inoculation of Mycobacterium paratuberculosis to guinea pigs and cattle. Am. J. Vet. Res. 23: 676-678.

18. Monaghan, M. L. and Hannan, J. 1983. Abattoir survey of bovine kidney disease. Vet. Rec. 113: 55-57.

19. Moravkova, M., Hlozek, P., Beran, V., Pavlik, I., Preziuso, S., Cuteri, V. and Bartos, M. 2008. Strategy for the detection and differentiation of Mycobacterium avium species in isolates and heavily infected tissues. Res. Vet. Sci. 85: 257-264.

20. Möbius, P., Hotzel, H., Raßbach, A. and Köhler, H. 2008. Comparison of 13 single-rounded and nested PCR assays targeting IS900, SMav2, f57 and locus 255 for detection of Mycobacterium avium subsp. paratuberculosis. Vet. Microbiol. 126: 324-333.

21. Nielsen, S. S. 2008. Transitions in diagnostic tests used for detection of Mycobacterium avium subsp. paratuberculosis infections in cattle. Vet. Microbiol. 132: 274-282

22. Nielsen, S. S., Nielsen, K. K., Huda, A., Condron, R. and Collins, M. T. 2001. Diagnostic techniques for paratuberculosis. pp. 5-17. In: Bulletin-International Dairy Federation 362/ 2001, International Dairy Federation, Brussels.

23. Nielsen, S. S. and Toft, N. 2008. Ante mortem diagnosis of paratuberculosis: a review of accuracies of ELISA, interferon- $\gamma$ 
assay and faecal culture techniques. Vet. Microbiol. 129: 217 235.

24. Pagl, R., Aurich, J. E., Müller-Schlösser, F., Kankofer, M. and Aurich, C. 2006. Comparison of an extender containing defined milk protein fractions with a skim milk-based extender for storage of equine semen at $5^{\circ} \mathrm{C}$. Theriogen 66: 1115-1122.

25. Palmer, C. W. 2005. Welfare aspects of theriogenology: investigating alternatives to electroejaculation of bulls. Theriogen 64: 469-479.

26. Pavlik, I., Matlova, L., Bartl, J., Svastova, P., Dvorska, L. and Whitlock, R. 2000. Parallel fecal and organ Mycobacterium avium subsp. paratuberculosis culture of different productivity types of cattle. Vet. Microbiol. 77: 309-324.

27. Philpott, M. 1993. The danger of disease transmission by artificial insemination and embryo transfer. Br. Vet. J. 149: 339369.

28. Revell, S. G., Chasey, D., Drew, T. W. and Edwards, S. 1988. Some observations on the semen of bulls persistently infected with bovine virus diarrhoea virus. Vet. Rec. 123: 122-125.

29. Sivakumar, P., Tripathi, B. N. and Singh, N. 2005. Detection of Mycobacterium avium subsp. paratuberculosis in intestinal and lymph node tissues of water buffaloes (Bubalis bubalis) by
PCR and bacterial culture. Vet. Microbiol. 108: 263-270.

30. Slana, I., Kralik, P., Kralova, A. and Pavlik, I. 2008. On-farm spread of Mycobacterium avium subsp. paratuberculosis in raw milk studied by IS 900 and $F 57$ competitive real time quantitative PCR and culture examination. Int. J. Food Microbiol. 128: 250-257.

31. Slana, I., Paolicchi, F., Janstova, B., Navratilova, P. and Pavlik, I. 2008. Detection methods for Mycobacterium avium subsp. paratuberculosis in milk and milk products: a review. Vet. Med. 53: 283-306.

32. Tasara, T. and Stephan, R. 2005. Development of an F57 sequence-based real time PCR assay for detection of Mycobacterium avium subsp. paratuberculosis in Milk. Ap. Env. Microbiol. 71: 5957-5968.

33. Whipple, D. L., Callihan, D. R. and Jarnagin, J. L. 1999. Cultivation of M. paratuberculosis from bovine faecal specimens and a suggested standardised procedure. J. Vet. Diagn. Invest. 3: $368-373$.

34. Whitlock, R. 1996. Johne's disease. pp. 899-904. In: Large Animal Internal Medicine, 2nd ed. (Smith, B. S. ed.), Mosby, St. Louis. 